OPEN ACCESS

Edited by:

Nan Kang,

Northwestern Polytechnical

University, China

Reviewed by:

Mehdi Derradji,

Polytechnic School of Algiers, Algeria

Yuanqing $\mathrm{Li}$,

Chongqing University, China

*Correspondence:

Pengfei Lei

pengfeilei@csu.edu.cn

Yihe Hu

csuhuyihe@163.com

${ }^{+}$These authors have contributed equally to this work

Specialty section:

This article was submitted to

Polymeric and Composite Materials,

a section of the journal

Frontiers in Materials

Received: 25 September 2020

Accepted: 07 January 2021

Published: 23 February 2021

Citation:

Xiong Z, Liu W, Qian H, Lei T, He X

Hu Y and Lei P (2021) Tantalum

Nanoparticles Reinforced PCL

Scaffolds Using Direct 3D Printing for

Bone Tissue Engineering.

Front. Mater. 8:609779.

doi: 10.3389/fmats.2021.609779

\section{Tantalum Nanoparticles Reinforced PCL Scaffolds Using Direct 3D Printing for Bone Tissue Engineering}

\author{
Zixuan Xiong ${ }^{1,2,3 \dagger}$, Wenbin $\mathrm{Liu}^{1,2 \dagger}$, Hu Qian ${ }^{1,2}$, Ting $\mathrm{Lei}^{1,2}, \mathrm{Xi} \mathrm{He}^{1,2}$, Yihe $\mathrm{Hu}^{1,2 *}$ and \\ Pengfei Lei ${ }^{1,2 *}$
}

${ }^{1}$ Department of Orthopedics, Xiangya Hospital of Central South University, Changsha, China, ${ }^{2}$ Hunan Engineering Research Center of Biomedical Metal and Ceramic Implants, Changsha, China, ${ }^{3}$ Xiangya School of Medicine, Central South University, Changsha, China

Polycarbonate (PCL) has been widely used in tissue engineering, but its hydrophobicity and low biological activity limit its further promotion and application. By adding nanoparticles, the hydrophilicity and biological activity of PCL can be improved. In this study, different amounts of Ta (1-10\%wt) were added to PCL, and then their mechanical and biological properties were studied in vitro. XRD found that 5\%Ta-PCL has the highest crystallinity. At the same time, cell experiments CCK8, cell adhesion, osteogenic differentiation, and osteogenesis related gene expression showed that Ta can enhance the mechanical and biological properties of PCL, while 5\% Ta-PCL showed the best mechanical and biological properties. This composite of tantalum and PCL could have a clinical potential for orthopedic implants.

Keywords: polycarbonate(PCL), tantalum, bone tissue engineering, osteogenesis, 3D printing

\section{INTRODUCTION}

Every year, millions of people suffer from massive bone defects due to various reasons, such as tumors, trauma, and infections, resulting in an increasing clinical demand for bone defect implants (Bhattacharjee et al., 2017; Wang and Yeung, 2017). Autologous bone transplantation is the golden standard for clinical treatment of bone defects, but its source is limited, and it will increase the risk of fracture at the donor site, which greatly limits its clinical application in the treatment of large bone defects (Tang et al., 2016; Ji et al., 2020). Xenogeneic bone can also be used to treat large bone defects, but there is a risk of immune response, disease transmission and treatment safety (Ma et al., 2018). Therefore, scaffold-based tissue engineering technology has attracted more and more people's interest.

Polycarbonate (PCL) is a biodegradable polyester certified by the FDA (Fedorová et al., 2015; Felice et al., 2018). Which is non-toxic and harmless to tissues and has good biocompatibility. It is often used as a scaffold in bone tissue engineering (Masoudi Rad et al., 2017; Wang et al., 2018; Ji et al., 2020). At the same time, the degradation products, $\mathrm{CO}_{2}$, and $\mathrm{H}_{2} \mathrm{O}$, are no harmful substances (Bittner et al., 2019). However, the hydrophobic surface together with the lack of its strength and biological activity result in the unfavorable properties of PCL for cell adhesion (Heo et al., 2019). Therefore, how to improve the strength and hydrophilicity of PCL is a hot spot in current research.

As a transition metal element, tantalum has good physical and chemical properties such as high melting point, high strength, wear resistance and corrosion resistance (Niinomi et al., 2012; Torstrick et al., 2016). Among them, the good corrosion resistance is due to the $\mathrm{Ta} 2 \mathrm{O} 5$ film formed on the surface. Tantalum is a metal material with excellent biocompatibility, whose porous structure can 

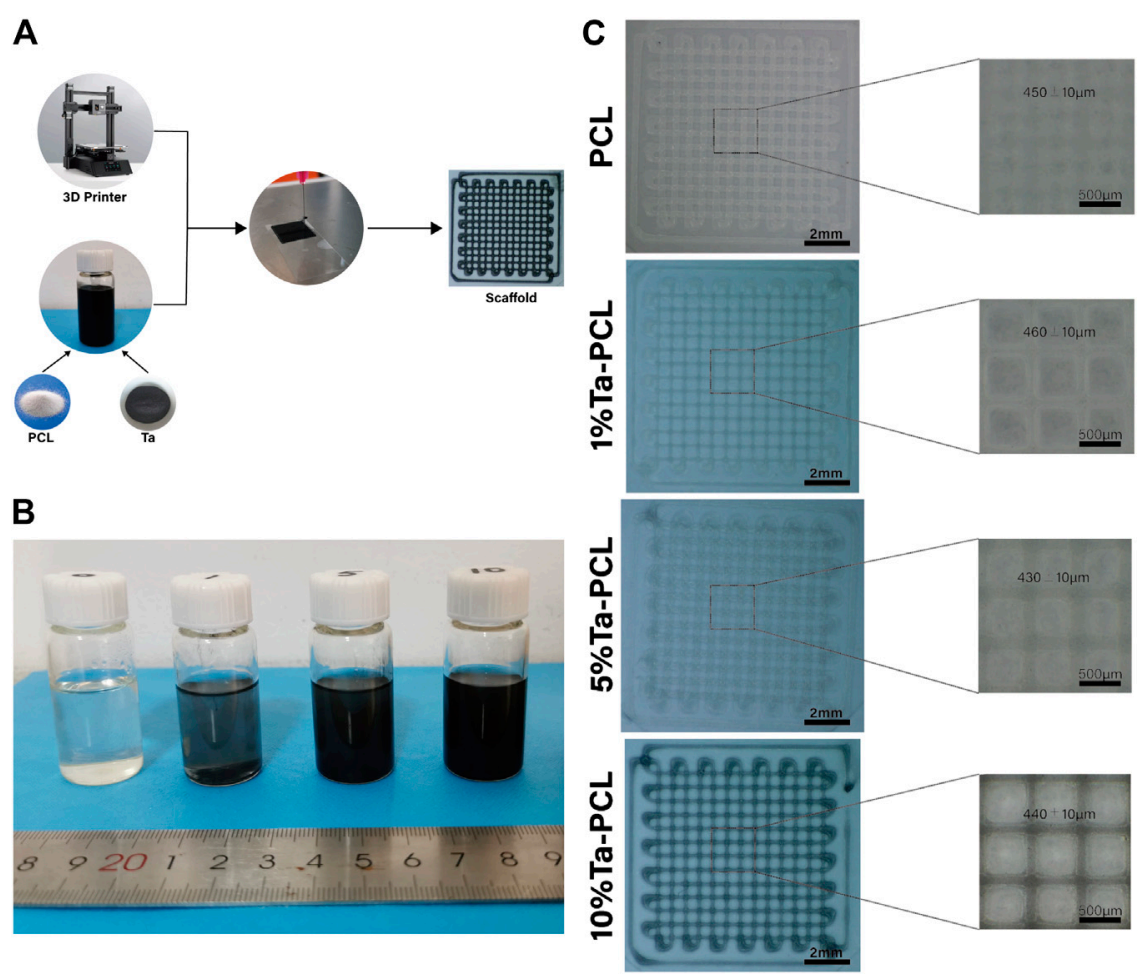

FIGURE 1|The characterization of Ta/PCL scaffold. (A) The synthesis process of Ta/PCL. (B) The general view of Ta/PCL solution. (C) Front view of the structure of $\mathrm{Ta} / \mathrm{PCL}$.

mimic the trabecular bone structure of normal bone tissue (Levine et al., 2006). At the same time, tantalum and nanotantalum have also been proved to have the characteristics of promoting the osteogenic differentiation of mesenchymal stem cells (Mohandas et al., 2014; Wang et al., 2015; Kang et al., 2017). Nano-tantalum have a larger specific surface area than tantalum metal which can provide stronger biological activity. Zhu Hao et al. used tantalum nanoparticles combined with PEEK had been shown to enhance the mechanical properties and osteogenic activity of PEEK (Zhu et al., 2019). Park et al. demonstrated Ta-implanted PLA could significantly enhanced osseointegration and osteogenesis compared with bare PLA (Park et al., 2019). Since tantalum nanoparticles are stable and biocompatible, they might strengthen the properties of the hydrophilic and mechanical of PCL, and potentially alter the properties of PCL for better prospect in orthopedic use.

In this study, we first used nano-tantalum blended PCL with $3 \mathrm{D}$ printing to enhance PCL, and fabricated Ta-PCL doped with different proportions of tantalum nanoparticles (1, 5 and $10 \% w t)$ to study the effect of the 3D Ta-PCL scaffold on the osteogenic differentiation of mesenchymal stem cells in vitro. Our hypothesis is that $\mathrm{Ta}$ nanoparticles can enhance the crystallinity and hydrophilic properties of PCL (Supplementary Figure S3) and further enhance the osteogenic differentiation ability of mesenchymal stem cells. The osteogenic differentiation ability of 3D Ta-PCL was tested by RT-PCR and COL I staining.

\section{MATERIALS AND METHODS}

\section{Sample Fabrication and Preparation}

The Polycarbonate particles (Sigma, MW=80,000, United Kingdom) are thoroughly mixed with tantalum nanoparticles (Dk Nano Technology Co., LTD., Beijing, China Size $=50 \mathrm{~nm})($ Supplementary Figure S2), and the mixture was added to a solution composed by dichloromethane and absolute ethanol. The proportion of tantalum nanoparticles is controlled at 1,5 and $10 \mathrm{wt} \%$. The volume ratio of dichloromethane to absolute ethanol is controlled at 2:3. Pure PCL particles were used as a control group. For each group, according to a specific procedure, a mechanical extrusion method is used to form the material. In short, the mixed material is first added to the mixed solution, and then extruded through a fine pinhole to quickly evaporate the solvent, leaving only the solid powder to form the stent (Figure 1A).

We designed several programs in advance. Small square samples $\left(10 \times 10 \times 0.3 \mathrm{~mm}^{3}\right)$ were used in 24 -well culture plates for surface characterization and in vitro studies. Large square samples $\left(13 \times 13 \times 0.3 \mathrm{~mm}^{3}\right)$ were used for RNA expression related tests. In order to remove the debris on the samples and the microorganisms in the air stained during printing, all samples were soaked in medical ethanol for $8 \mathrm{~h}$ and then irradiated with ultraviolet light for $1 \mathrm{~h}$. Then all samples were washed 5 times with phosphate buffered saline (PBS). 


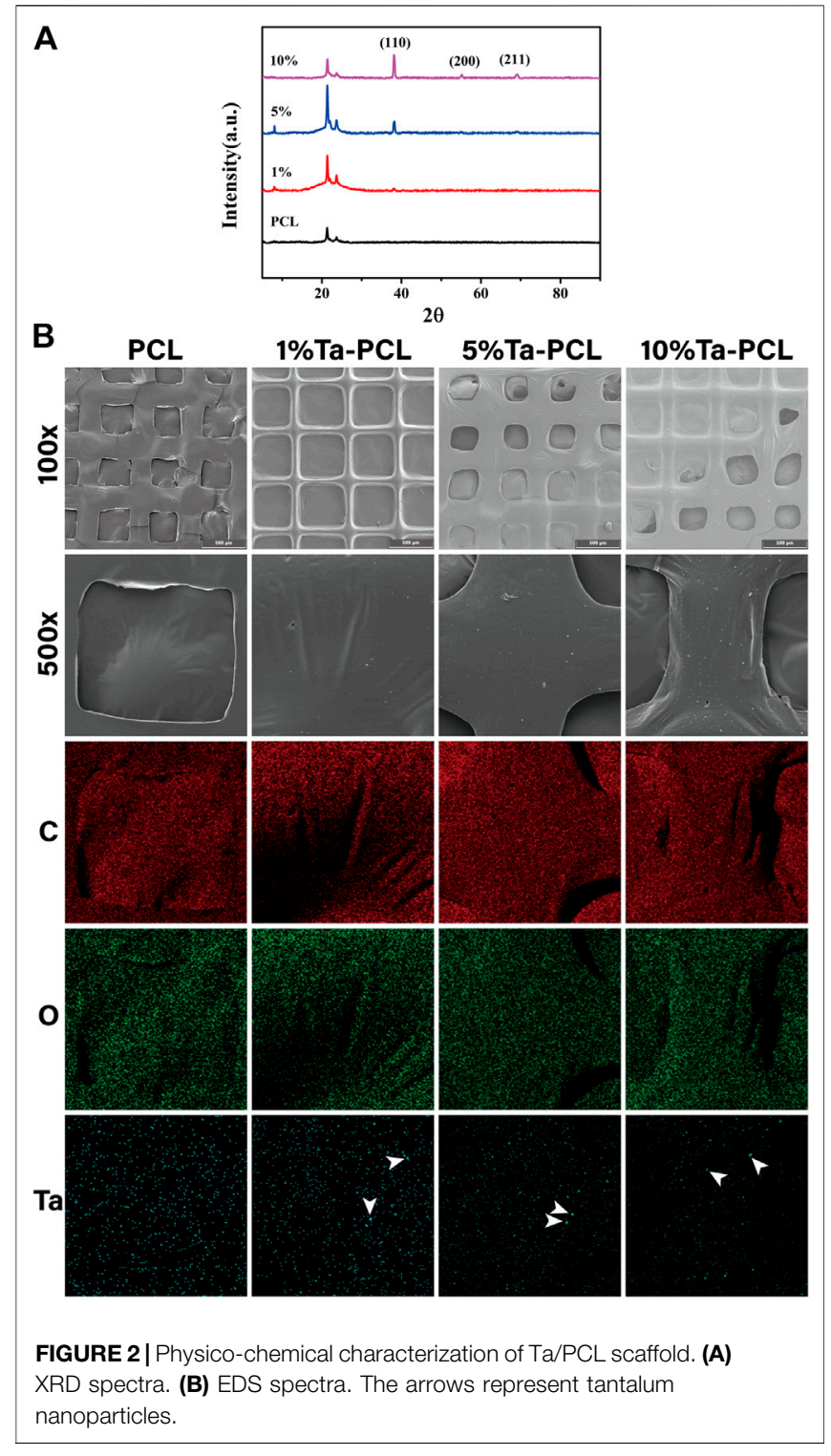

\section{Material Properties}

The surface morphology was examined by scanning electron microscope (Quanta-200, United States) after sputtered with $5 \mathrm{~nm}$ of platinum coating (Gressington 308R,United Kingdom). The images were taken in different magnifications when the acceleration voltage is $10 \mathrm{kV}$, after that, the energy-dispersive $\mathrm{X}$-ray spectroscopy (EDS) was performed.

\section{In vitro Studies \\ Cell Culturing}

The bone marrow mesenchymal stem cells of rats (rBMSCs) are used for in vitro research (Song et al., 2017). The cells were cultured in DMEM/F-12 essential medium (Gibco, United States) in standard culture environment, such as $37^{\circ} \mathrm{C}$ humid environment and $5 \% \mathrm{CO}_{2}$. The medium contains $10 \%$ fetal bovine serum and $1 \%$ antibiotics (50 IU penicillin/ml and $50 \mu \mathrm{g}$ streptomycin/ml) and it was changed every three days,

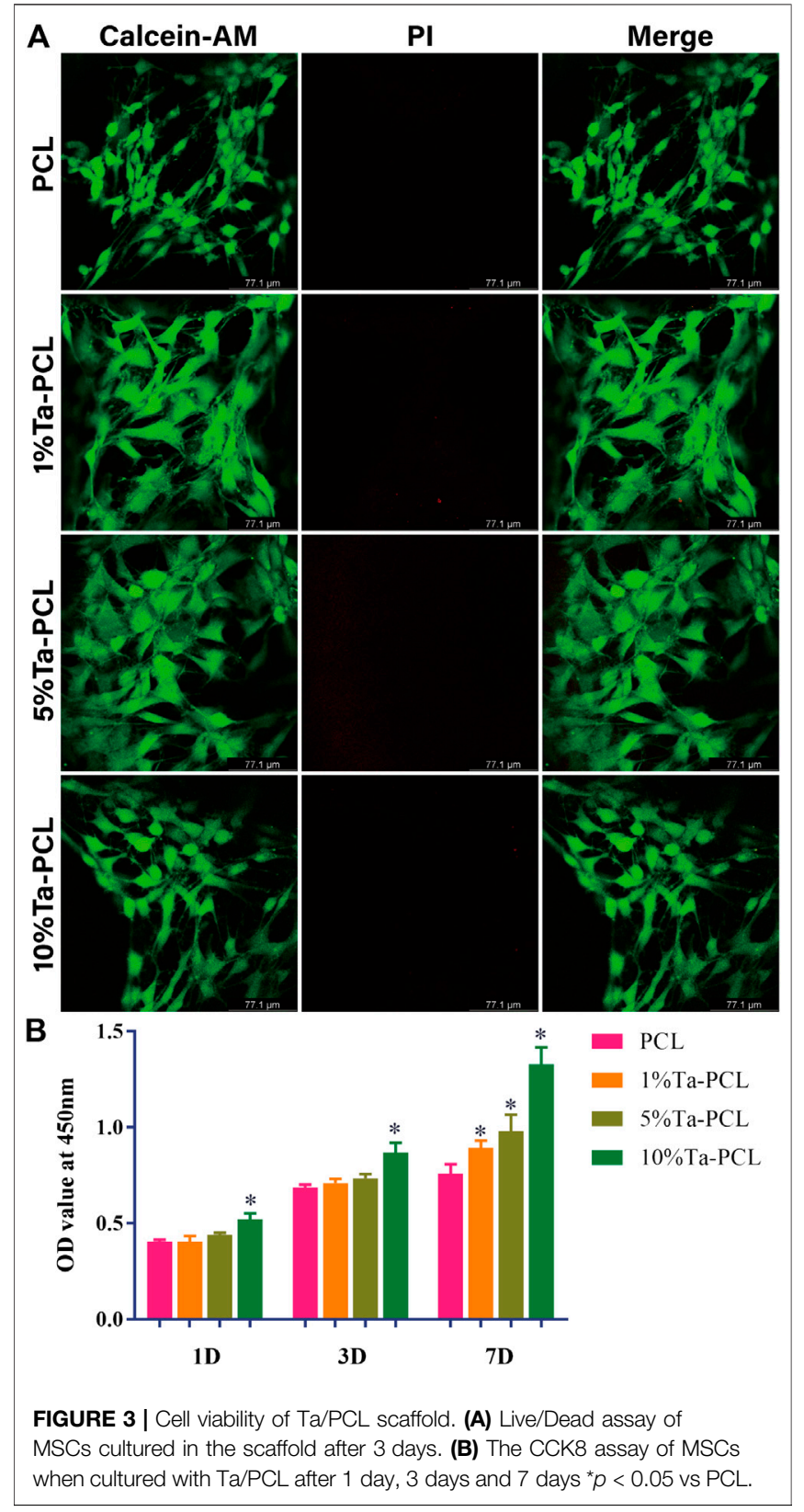

the cells were passaged when the coverage of the medium reached $90 \%$.

\section{Cell Adhesion}

The rBMSCs were seeded onto the surface of the samples $(10 \times$ $10 \times 0.3 \mathrm{~mm}^{3}$ ) at a density of $1 \times 104 /$ well. After culturing for $24 \mathrm{~h}$ respectively, the medium was removed and samples were rinsed with PBS for 3 times, together with the fixation with $4 \%$ paraformaldehyde in PBS. Samples were then stained with phalloidin and DAPI. Images were taken under confocal scanning microscopy (Leica TCS SP8 X, Germany). For SEM characterization, the samples were dehydrated by gradient ethanol and dried in the air. 


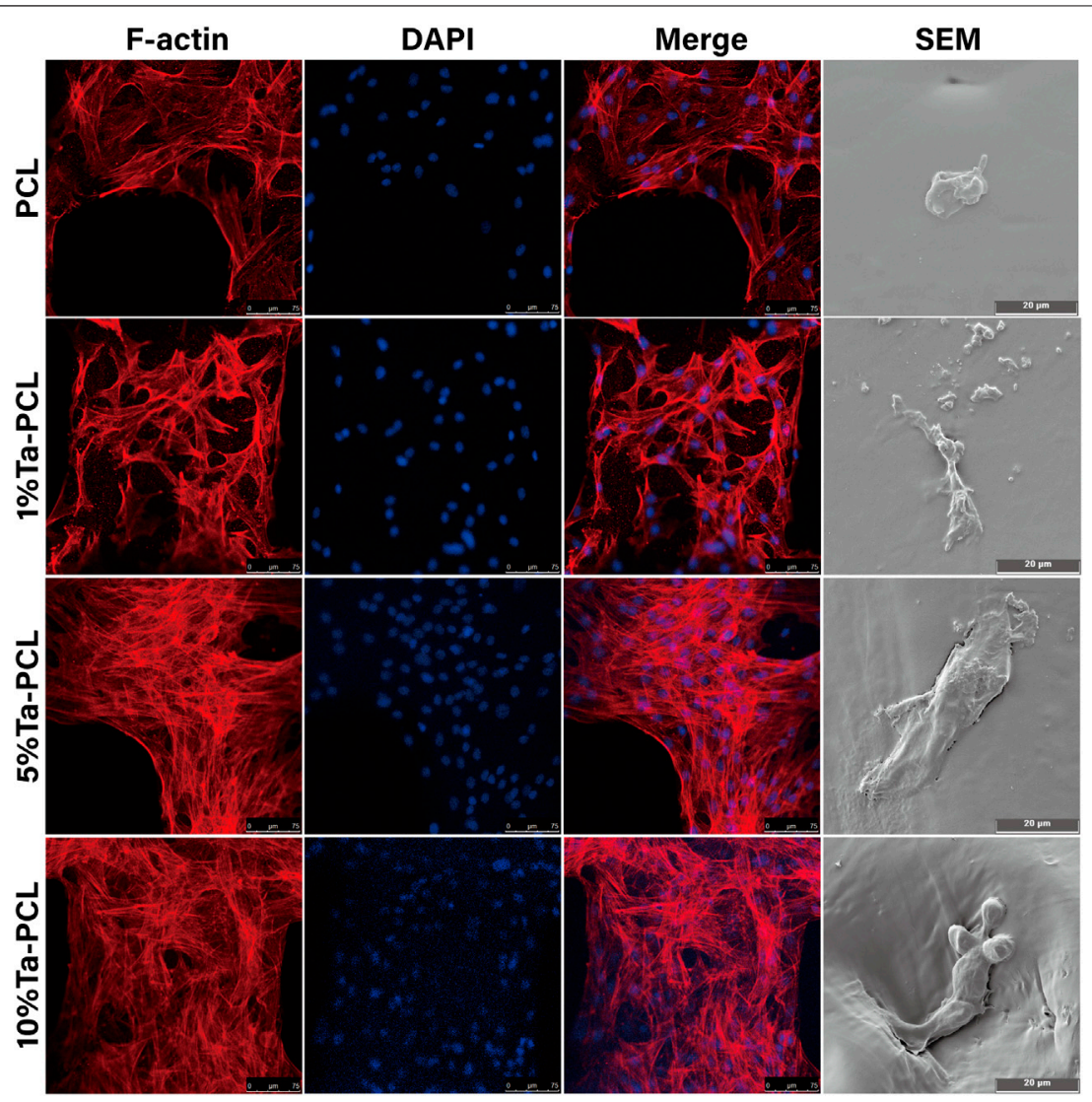

FIGURE 4 | Cell adhesion of Ta/PCL scaffold. Cytoskeleton of MSCs cultured on the scaffold for 5 days.

\section{Osteogenesis Related Gene Expression}

The expression of osteogenesis-related genes was analyzed by realtime polymerase chain reaction (qPCR). Briefly, rBMSCs were seeded with a density of $2 \times 104 /$ well and cultured for 7 days, Total RNA was extracted using Trizol reagent (Invitrogen, United States), Subsequently, nanodrop 2000 was used to obtain the concentration of RNA, after which the reverse transcription using a ReverTra Ace ${ }^{\circledR}$ qPCR RT Kit (Toyobo, Japan). The experiment was carried with CFX Connect Real-Time PCR Detection System (BIO$\mathrm{RAD}$, United States) , $\beta$-actin was used as a housekeeping gene, and the relative quantification of mRNA (ALP, OCN and COL I) was determined with $2-\triangle \triangle \mathrm{Ct}$ method. The primers are ALP: Forward primer sequence $\left(5^{\prime}-3^{\prime}\right)$ : GCACAACATCAAGGACATCG; Reverse primer sequence $\left(5^{\prime}-3^{\prime}\right)$ : TCAGTTCTGTTCTTGGGGTACAT; COL I: Forward primer sequence $\left(5^{\prime}-3^{\prime}\right)$ : GAAGACCTGGCG AGAGAGGA; Reverse primer sequence $\left(5^{\prime}-3^{\prime}\right)$ : TCAATCCAT CCAGACCGTTG. OCN: Forward primer sequence $\left(5^{\prime}-3^{\prime}\right)$ : GCC CTGACTGCATTCTGCCTCT; Reverse primer sequence $\left(5^{\prime}-3^{\prime}\right)$ : TCACCACCTTACTGCCCTCCTG.

\section{Statistical Analysis}

Data were expressed as means standard deviations (SD) from experiments performed in quintuplicate. Statistical analysis was performed using the one-way analysis of variance (ANOVA) with Tukey-Kramer Multiple comparison post-test using GraphPad
Instant Software (GraphPad Prism Software, Inc. United States) with $p<0.05$ being considered to be statistically significant.

\section{RESULT AND DISCUSSION}

\section{Characterization of Ta-PCL Scaffolds}

We first aimed to obtain the Ta-PCL inks with perfect Printability, and then $3 \mathrm{D}$ printed different proportions of TA-PCL onto a glass slide through an extrusion printer. It could be seen from the general view (Figures 1B, C) that the printed shape of 1, 5 and $10 w t \%$ Ta-PCL can be formed regularly. With the increasing proportions of $\mathrm{Ta}$ nanoparticles, the color of the scaffolds gradually became black. The pore size and porosity of the scaffold are important influencing factors for bone tissue regeneration. A proper pore size can promote the formation of blood vessels and the flow of nutrients to promote bone regeneration. The pore size of the scaffold was analyzed. The pore size of the PCL scaffold was $450 \pm 10 \mathrm{um}, 1 \% \mathrm{Ta}$-PCL scaffold pore diameter is $460 \pm 10 \mathrm{um}, 5 \% \mathrm{Ta}-\mathrm{PCL}$ scaffold pore diameter was $430 \pm 10 \mathrm{um}, 10 \% \mathrm{Ta}-\mathrm{PCL}$ pore diameter was $440 \pm$ $10 \mathrm{um} .300-500 \mathrm{um}$ is the best pore diameter to promote osteogenesis, and all scaffolds' pore were designed to be in this range (Ma et al., 2020), the size of the pore changes slightly between different scaffold which may related to the error of the printer. At 


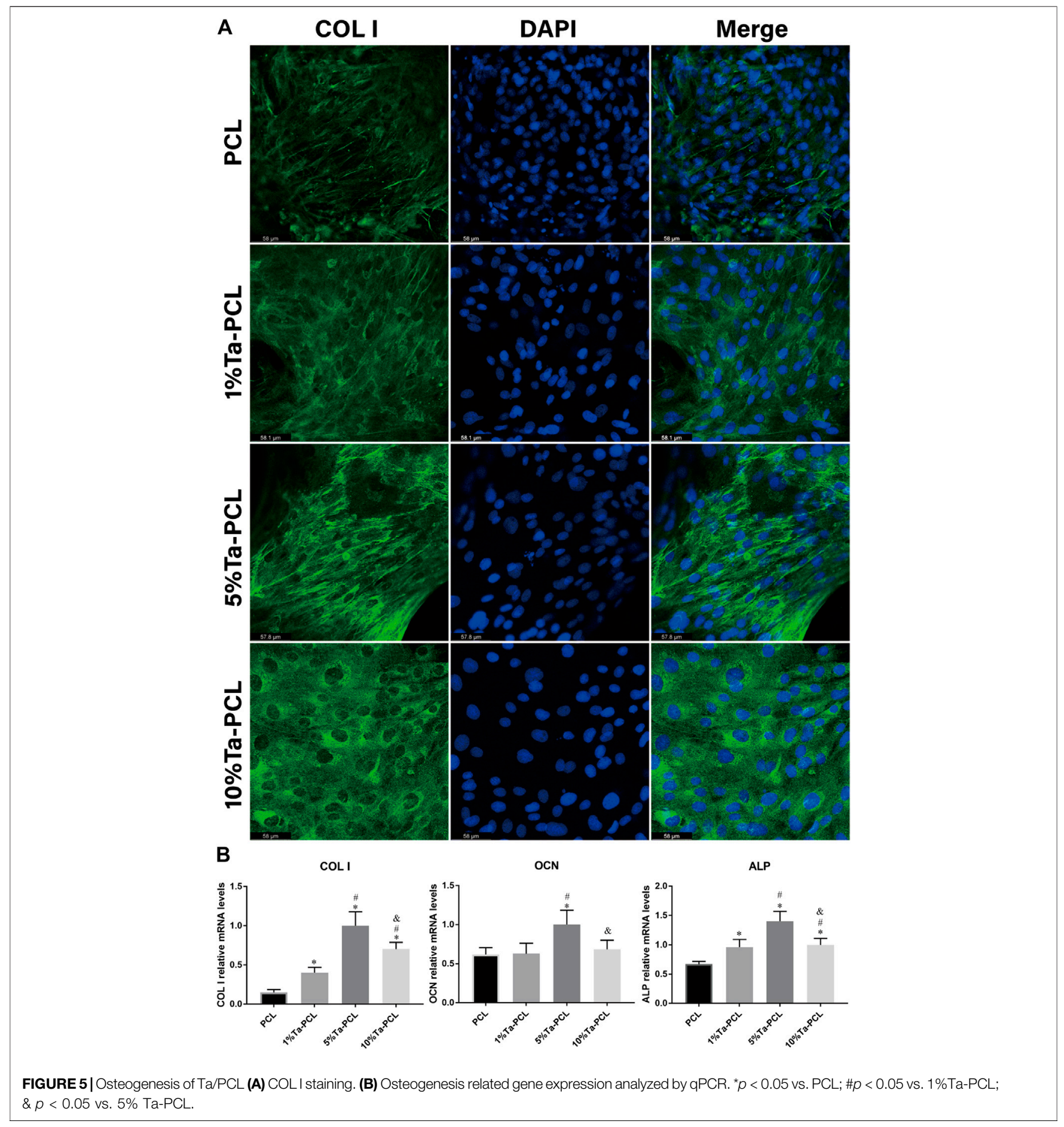

the same time, the tantalum nanoparticles were found uniformly dispersed in the PCL through the SEM rather than agglomerated together (Supplementary Figure S1), which is basically consistent with the researches of others. To further confirm the presence of Ta nanoparticles in the scaffold, EDS was performed. The EDS results show that there are characteristic peaks of tantalum on the surface of PCL doped with different contents of tantalum.
In order to further explored the crystallization situation inside the Ta-PCL, XRD was performed. Figure $2 \mathrm{~A}$ shows that the XRD patterns of Ta-PCL scaffolds and pure PCL scaffolds with different contents. In the XRD pattern, Ta shown three typical characteristic peaks at $2 \theta=38.2^{\circ}, 55.1^{\circ}$ and $69.1^{\circ}$. The corresponding crystal planes were (110) (200) and (211), which were similar to the diffraction peaks of standard Ta. 
From the XRD pattern, pure PCL fibers shown two typical diffraction peaks at $2 \theta=21.4^{\circ}$ and $24.3^{\circ}$. In the composite scaffolds, with the increase of $\mathrm{Ta}$ content, the diffraction intensity of the PCL characteristic peak first increased and then decreased, indicating that the crystallinity of PCL was improved after the usage of $\mathrm{Ta}$, and the crystallinity of TaPCL scaffold reached the summit at $5 \mathrm{wt} \%$. That is because of The non-crystalline region of PCL is made up of the polymer chain segment, and the nanoparticles and the chain segment will work together to reduce the polymer base's free body product, allowing the chain to be arranged in the non-crystalline zone, so the crystallization increase, and when the amount of nanoparticles increases, it also damage the chain failure of the original crystallization zone and the crystallization degree decreases. Based on the results above, we had successfully prepared 3D printed Ta-PCL scaffold with different contents.

\section{Cell Compatibility of the Ta-PCL Scaffold}

In order to evaluate the biocompatibility of the scaffold, we conducted CCK8 and Live/dead staining to evaluate the cytotoxicity of the scaffold. As was shown in Figure 3, the addition of $\mathrm{Ta}$ nanoparticles could promote the proliferation of mesenchymal stem cells, and with the increasing content of tantalum, 10\% Ta-PCL shown to be the best cell proliferation rate. Live/dead staining also shown that $\mathrm{PCL}, 1,5$ and $10 \% \mathrm{Ta}-$ PCL were all non-toxic and biocompatible.

Initial cell adhesion and spreading on biomaterials, the first phase of the cell material interaction, is a key step in the regulation of the cell proliferation and differentiation that subsequently follows. In order to evaluate the adhesion performance of Ta-PCL to mesenchymal stem cells, they were planted on the scaffold for 3 days Figure 4 showed that PCL had poor surface cell morphology due to its poor hydrophilicity. The cell morphology on $1 \%$ Ta-PCL was more stretched, while the cells on 5 and $10 \%$ TaPCL were fully stretched with a large number of pseudopods being observed, while 5\% Ta-PCL has more stretched cell morphology and better cell adhesion performance.

\section{Osteogenesis of the Ta-PCL Scaffold}

Col I expression in BMSCs for calcium nodules produced by mineralization in the late stage of osteogenic differentiation, which may further promote the mineralization in the later stage of osteogenic differentiation (Wang et al., 2021). Co-cultivation of mesenchymal stem cells with 3D printed Ta-PCL scaffolds 7 days later, Figure 5A showed that the COL I expression of the Ta-PCL scaffold was higher than that of the PCL scaffold alone, indicating that the addition of Ta can promote the secretion of collagen, which may further promote the formation of calcium nodules. 5\% Ta-PCL had the highest expression of COL I, followed by 10 and 1\%Ta-PCL. This was basically consistent with the research of others.

To further examined the influence of the Ta-PCL on the differentiation of osteogenic at the molecular level, The expression of osteogenic marker genes such as collagen I (Col I), alkaline phosphatase (ALP), osteocalcin (OCN) and in BMSCs was assayed using quantitative real-time PCR after 7 days of osteogenic induction culture. As is shown in Figure 5B,.ALP and COL I are characteristic markers of osteogenic differentiation, which can promote cell osteogenic mineralization and the formation of calcium nodules (Chen et al., 2020; Shen et al., 2020). Comparing with PCL, 5\% Ta-PCL shown the highest ALP expression. It also had the highest COL I expression, which was perfectly consistent with immunofluorescence staining (Figure $\mathbf{5 A}$ ). OCN is a characteristic marker of late osteogenesis (Rasi Ghaemi et al., 2016; Sun et al., 2019). In Figure 5B, tantalum nanoparticle could enhance the expression of OCN compared to PCL, with the 5\%Ta-PCL having the highest gene expression compared to 1 and $10 \% \mathrm{Ta}-$ PCL. Generally speaking, mesenchymal stem cells had the highest osteogenic differentiation potential in 5\% Ta-PCL in terms of gene expression and protein expression.

\section{CONCLUSION}

In summary, we obtained a scaffold with good pore structure and porosity by $3 \mathrm{D}$ printing the mixture of PCL and Ta nanoparticles. SEM and XRD showed that Ta nanoparticles were evenly distributed in PCL. The 5\% Ta-PCL composite scaffold had the best crystallinity. At the same time, CCK 8 and live/dead staining found that the addition of Ta nanoparticles could promote the proliferation and osteogenic differentiation of mesenchymal stem cells while it had nothing to do with the activities of cells. In addition, RT-PCR found that 5\% Ta-PCL has the best osteoinductive property compared to 1 and 10\% Ta-PCL. Altogether, these results shown that the $3 \mathrm{D}$ printed Ta-PCL scaffold has a good application prospect in bone tissue engineering.

\section{DATA AVAILABILITY STATEMENT}

The original contributions presented in the study are included in the article/Supplementary Material, further inquiries can be directed to the corresponding authors.

\section{ETHICS STATEMENT}

The animal study was reviewed and approved by the Ethical Review Board at the Xiangya Hospital of Central South University (Changsha, China).

\section{AUTHOR CONTRIBUTIONS}

The manuscript was written through contributions of all authors. All authors have given approval to the final version of the manuscript. LW and XZ contributed equally.

\section{FUNDING}

This study was supported by the Natural Science Foundation of Hunan Province, China (Grant No. 2018JJ3844 and 2019JJ40499), the Scientific Research Project of Health and Family Planning 
Commission of Hunan Province, China (Grant No. B2019188), the Science and technology Innovation Leading Project for High-tech Industry of Hunan Province (Grant No. 2020SK2008), the Major science and technology projects of Changsha City (Grant No. kh2003016), the Young Science Foundation of Xiangya Hospital Central South University (Grant No. 2017Q07), the Postdoctoral Research Program of Xiangya Hospital Central South University (Grant No. 223551). National key research and development project (2016YFC1100605 and 2018YFB1105504), Natural Science Foundation of China (Grant No. 82002277 and

\section{REFERENCES}

Bhattacharjee, P., Kundu, B., Naskar, D., Kim, H. W., Maiti, T. K., Bhattacharya, D., et al. (2017). Silk scaffolds in bone tissue engineering: an overview. Acta Biomater. 63, 1-17. doi:10.1016/j.actbio.2017.09.027

Bittner, S. M., Smith, B. T., Diaz-Gomez, L., Hudgins, C. D., Melchiorri, A. J., Scott, D. W., et al. (2019). Fabrication and mechanical characterization of 3D printed vertical uniform and gradient scaffolds for bone and osteochondral tissue engineering. Acta Biomater. 90, 37-48. doi:10.1016/j.actbio.2019.03.041

Chen, G., Kong, P., Jiang, A., Wang, X., Sun, Y., Yu, T., et al. (2020). A modular programmed biphasic dual-delivery system on $3 \mathrm{D}$ ceramic scaffolds for osteogenesis in vitro and in vivo. J. Mater. Chem. B, 8, 9697. doi:10.1039/c9tb02127b

Fedorová, P., Srnec, R., Pěnčík, J., Dvořák, M., Krbec, M., and Nečas, A. (2015). [Intra-articular reinforcement of a partially torn anterior cruciate ligament (ACL) using newly developed UHMWPE biomaterial in combination with Hexalon ACL/PCL screws: ex-vivo mechanical testing of an animal knee model]. Acta Chir. Orthop. Traumatol. Cech. 82 (3), 222-228.

Felice, B., Sánchez, M. A., Socci, M. C., Sappia, L. D., Gómez, M. I., Cruz, M. K., et al. (2018). Controlled degradability of PCL-ZnO nanofibrous scaffolds for bone tissue engineering and their antibacterial activity. Mater. Sci. Eng. C 93, 724-738. doi:10.1016/j.msec.2018.08.009

Heo, S. Y., Ko, S. C., Oh, G. W., Kim, N., Choi, I. W., Park, W. S., et al. (2019). Fabrication and characterization of the 3D-printed polycaprolactone/fish bone extract scaffolds for bone tissue regeneration. J. Biomed. Mater. Res. B Appl. Biomater. 107 (6), 1937-1944. doi:10.1002/jbm.b.34286

Ji, X., Yuan, X., Ma, L., Bi, B., Zhu, H., Lei, Z., et al. (2020). Mesenchymal stem cellloaded thermosensitive hydroxypropyl chitin hydrogel combined with a threedimensional-printed poly( $\varepsilon$-caprolactone)/nano-hydroxyapatite scaffold to repair bone defects via osteogenesis, angiogenesis and immunomodulation. Theranostics 10 (2), 725-740. doi:10.7150/thno.39167

Kang, C., Wei, L., Song, B., Chen, L., Liu, J., Deng, B., et al. (2017). Involvement of autophagy in tantalum nanoparticle-induced osteoblast proliferation. Int. J. Nanomed. 12, 4323-4333. doi:10.2147/ijn.S136281

Levine, B. R., Sporer, S., Poggie, R. A., Della Valle, C. J., and Jacobs, J. J. (2006). Experimental and clinical performance of porous tantalum in orthopedic surgery. Biomaterials 27 (27), 4671-4681. doi:10.1016/j.biomaterials.2006.04.041

Ma, H., Feng, C., Chang, J., and Wu, C. (2018). 3D-printed bioceramic scaffolds: from bone tissue engineering to tumor therapy. Acta Biomater. 79, 37-59. doi:10.1016/j.actbio.2018.08.026

Ma, L., Cheng, S., Ji, X., Zhou, Y., Zhang, Y., Li, Q., et al. (2020). Immobilizing magnesium ions on $3 \mathrm{D}$ printed porous tantalum scaffolds with polydopamine for improved vascularization and osteogenesis. Mater. Sci. Eng. C Mater. Biol. Appl. 117, 111303. doi:10.1016/j.msec.2020.111303

Masoudi Rad, M., Nouri Khorasani, S., Ghasemi-Mobarakeh, L., Prabhakaran, M. P., Foroughi, M. R., Kharaziha, M., et al. (2017). Fabrication and characterization of twolayered nanofibrous membrane for guided bone and tissue regeneration application. Mater. Sci. Eng. C Mater. Biol. Appl. 80, 75-87. doi:10.1016/j.msec.2017.05.125

Mohandas, G., Oskolkov, N., McMahon, M. T., Walczak, P., and Janowski, M. (2014). Porous tantalum and tantalum oxide nanoparticles for regenerative medicine. Acta Neurobiol. Exp. 74 (2), 188-196.

Niinomi, M., Nakai, M., and Hieda, J. (2012). Development of new metallic alloys for biomedical applications. Acta Biomater. 8 (11), 3888-3903. doi:10.1016/j. actbio.2012.06.037
81672656). Project Program of National Clinical Research Center for Geriatric Disorders (Xiangya Hospital, Grant No. 2020LNJJ15).

\section{SUPPLEMENTARY MATERIAL}

The Supplementary Material for this article can be found online at: https://www.frontiersin.org/articles/10.3389/fmats.2021.609779/ full\#supplementary-material.

Park, C., Seong, Y. J., Kang, I. G., Song, E. H., Lee, H., Kim, J., et al. (2019). Enhanced osseointegration ability of poly(lactic acid) via tantalum sputteringbased plasma immersion ion implantation. ACS Appl. Mater. Interfaces 11 (11), 10492-10504. doi:10.1021/acsami.8b21363

Rasi Ghaemi, S., Delalat, B., Cetó, X., Harding, F. J., Tuke, J., and Voelcker, N. H. (2016). Synergistic influence of collagen I and BMP 2 drives osteogenic differentiation of mesenchymal stem cells: a cell microarray analysis. Acta Biomater. 34, 41-52. doi:10.1016/j.actbio.2015.07.027

Shen, K., Tang, Q., Fang, X., Zhang, C., Zhu, Z., Hou, Y., et al. (2020). The sustained release of dexamethasone from $\mathrm{TiO}(2)$ nanotubes reinforced by chitosan to enhance osteoblast function and anti-inflammation activity. Mater. Sci. Eng. C Mater. Biol. Appl. 116, 111241. doi:10.1016/j.msec.2020.111241

Song, F., Jiang, D., Wang, T., Wang, Y., Lou, Y., Zhang, Y., et al. (2017). Mechanical stress regulates osteogenesis and adipogenesis of rat mesenchymal stem cells through PI3K/Akt/GSK-3. BioMed Res. Int. 2017, 6027402. doi:10.1155/2017/ 6027402

Sun, Q., Nakata, H., Yamamoto, M., Kasugai, S., and Kuroda, S. (2019). Comparison of gingiva-derived and bone marrow mesenchymal stem cells for osteogenesis. J. Cell Mol. Med. 23 (11), 7592-7601. doi:10.1111/jcmm.14632

Tang, W., Lin, D., Yu, Y., Niu, H., Guo, H., Yuan, Y., et al. (2016). Bioinspired trimodal macro/micro/nano-porous scaffolds loading rhBMP-2 for complete regeneration of critical size bone defect. Acta Biomater. 32, 309-323. doi:10. 1016/j.actbio.2015.12.006

Torstrick, F. B., Evans, N. T., Stevens, H. Y., Gall, K., and Guldberg, R. E. (2016). Do surface porosity and pore size influence mechanical properties and cellular response to PEEK? Clin. Orthop. Relat. Res. 474 (11), 2373-2383. doi:10.1007/s11999-016-4833-0

Wang, C., Lai, J., Li, K., Zhu, S., Lu, B., Liu, J., et al. (2021). Cryogenic 3D printing of dual-delivery scaffolds for improved bone regeneration with enhanced vascularization. Bioact. Mater. 6 (1), 137-145. doi:10.1016/j.bioactmat.2020. 07.007

Wang, Q., Zhang, H., Li, Q., Ye, L., Gan, H., Liu, Y., et al. (2015). Biocompatibility and osteogenic properties of porous tantalum. Exp. Ther. Med. 9 (3), 780-786. doi:10.3892/etm.2015.2208

Wang, T., Zhai, Y., Nuzzo, M., Yang, X., Yang, Y., and Zhang, X. (2018). Layer-bylayer nanofiber-enabled engineering of biomimetic periosteum for bone repair and reconstruction. Biomaterials 182, 279-288. doi:10.1016/j.biomaterials. 2018.08.028

Wang, W., and Yeung, K. W. K. (2017). Bone grafts and biomaterials substitutes for bone defect repair: a review. Bioact. Mater. 2 (4), 224-247. doi:10.1016/j. bioactmat.2017.05.007

Zhu, H., Ji, X., Guan, H., Zhao, L., Zhao, L., Liu, C., et al. (2019). Tantalum nanoparticles reinforced polyetheretherketone shows enhanced bone formation. Mater. Sci. Eng. C Mater. Biol. Appl. 101, 232-242. doi:10.1016/j.msec.2019.03.091

Conflict of Interest: The authors declare that the research was conducted in the absence of any commercial or financial relationships that could be construed as a potential conflict of interest.

Copyright (c) 2021 Xiong, Liu, Qian, Lei, He, Hu and Lei. This is an open-access article distributed under the terms of the Creative Commons Attribution License (CC BY). The use, distribution or reproduction in other forums is permitted, provided the original author(s) and the copyright owner(s) are credited and that the original publication in this journal is cited, in accordance with accepted academic practice. No use, distribution or reproduction is permitted which does not comply with these terms. 